\title{
Atuação do enfermeiro no gerenciamento dos leitos hospitalares na percepção dos profissionais de enfermagem
}

\author{
Carla Souza Fogaça1, Lays Adriane Sampaio Braga', Elisabete \\ Mesquita Peres de Carvalho ${ }^{2}$ e Leila Bernarda Donato Göttems ${ }^{1,2}$ \\ 1Universidade Católica de Brasília, Brasil | laysadriane01@gmail.com; \\ carlafogaca1@gmail.com | https://orcid.org/0000-0003-0478-7667; https://orcid.org/0000- \\ 0002-4707-5638 \\ ${ }^{2}$ Fundação de Ensino e Pesquisa em Ciências da Saúde, Brasil | \\ elisabete_mpc@yahoo.com.br, leila.gottems@gmail.com | https://orcid.org/0000-0002-5140- \\ 0237; https://orcid.org/0000-0002-2675-8085.
}

\begin{abstract}
Resumo: Os enfermeiros desempenham um papel importante no gerenciamento em tempo real dos leitos hospitalares disponíveis, de forma a promover seu uso eficiente. Objetivo: analisar a percepção dos enfermeiros sobre o processo de trabalho na gestão de leitos. Método: estudo descritivo, exploratório, de abordagem qualitativa. Participaram 17 enfermeiros que atuam na gestão de leitos e na assistência, identificados por meio da técnica de Bola de Neve em Redes Sociais Virtuais, com envio eletrônico do instrumento de coleta de dados, no período de 22 de agosto a 21 de outubro de 2020. A análise dos dados foi feita conforme Bardin: pré-análise, exploração do material e tratamento dos resultados, inferência e interpretação. Resultados: foi possível encontrar três núcleos de sentido que foram divididos em três categorias:1- perfil da equipe da Gestão de Leitos segundo a qualificação e experiência profissional; 2- atuação do enfermeiro na Gestão de Leitos; e 3 - dificuldades e facilidades nos processos de trabalho da Gestão de Leitos. Conclusão: foram observadas potencialidades e dificuldades na atuação do enfermeiro na gestão de leitos, configurando-se como uma área em expansão que requer regulamentação e aperfeiçoamento.
\end{abstract}

Palavras-chave: Gestão em saúde; Leitos hospitalares; Pesquisa Qualitativa.

\begin{abstract}
Nurses' performance in the management of hospital beds in the perception of nursing professionals

Abstract: Nurses play an important role in the real-time management of available hospital beds in order to promote their efficient use. Objective: to analyze nurses' perception of the work process in bed management. Method: a descriptive, exploratory study with a qualitative approach. Seventeen nurses who work in bed management and assistance participated, identified through the Snowball technique in Virtual Social Networks, with electronic sending of the data collection instrument, from August 22 to October 21, 2020. Data analysis carried out according to Bardin: pre-analysis, material exploration and treatment of results, inference, and interpretation. Results: it was possible to find three cores of meaning that were divided into three categories:1- profile of the Bed Management team according to qualification and professional experience; 2- performance of nurses in Bed Management; and 3 - difficulties and facilities in the work processes of Bed Management. Conclusion: potentials and difficulties in the nurse's performance in bed management were observed, taking shape in an expanding area that requires regulation and improvement.
\end{abstract}

Keywords: Health management; Hospital bed capacity; Qualitative Research.

\section{Introdução}

A atenção hospitalar tem sido, ao longo de décadas, um dos principais temas de debate acerca da assistência no Sistema Único de Saúde (SUS). Na prática, as instituições hospitalares que desenvolvem ações e serviços de saúde no âmbito do SUS agregam uma série de funções que as caracterizam como organizações mais complexas do setor Saúde, com densidade tecnológica específica, de caráter multiprofissional e interdisciplinar. Essas organizações são responsáveis pela assistência aos usuários com condições agudas ou crônicas, exigindo-se assistência contínua em regime de internação e ações que abrangem a promoção da saúde, a prevenção de agravos, o diagnóstico, o tratamento e a reabilitação (Brasil, 2017; Miranda, Mendes \& Silva, 2017). 
O processo de desenvolvimento das organizações hospitalares em busca de alternativas para a melhoria da qualidade de seus serviços tem sido motivado por um conjunto de fatores, como o aumento da demanda e o número limitado de leitos hospitalares (Wasgen, da Silva Terres \& Machado, 2019). A transição demográfica, a tripla carga de doenças e a diminuição do número de leitos hospitalares (Fundação Oswaldo Cruz, 2019) são questões pertinentes no que concerne atualmente à gestão dos serviços de saúde.

Nesse contexto, em dezembro de 2013, foi instituída a Política Nacional de Atenção Hospitalar (PNHOSP), por meio de Portaria, pelo Ministério da Saúde. De acordo com o Eixo Hospitalar dessa Política, o modelo de atenção contempla um conjunto de dispositivos de cuidado que assegura o acesso, a qualidade da assistência e a segurança do paciente. Dentre esses dispositivos, figura o gerenciamento de leitos, que consiste na otimização da utilização dos leitos, aumentando a rotatividade dentro de critérios técnicos, com vistas a ampliar a ocupação de leitos e otimizar a utilização da capacidade instalada, e melhorando o atendimento ao usuário (Brasil, 2013).

$\mathrm{Na}$ atualidade dos serviços públicos e privados, a gestão de leitos tem potencial para promover o uso dinâmico dos leitos hospitalares, por meio do aumento de rotatividade e monitoramento das atividades de Gestão da Clínica. Os enfermeiros desempenham um papel importante no gerenciamento em tempo real dos leitos hospitalares, de forma a promover um uso mais eficiente dos leitos disponíveis (Brasil, 2017). Assim, mais conhecimentos e habilidades clínicas são postas aos enfermeiros, permitindo a tomada de decisões, a destinação do paciente ao leito hospitalar, e conquistando um novo espaço de atuação, os quais levam a realizar diversas atividades nesse setor.

A atuação da enfermagem na regulação em saúde e, em especial, na gestão de leitos se constitui em atividades ancoradas na dimensão administrativo-gerencial, as quais também estão respaldadas na Lei do Exercício profissional (Brasil, 1986). Por ser uma atividade relativamente recente no âmbito do SUS, essa atuação tem sido pouco explorada na literatura específica da área.

Diante do exposto, este estudo tem como objetivo analisar a percepção dos enfermeiros sobre o processo de trabalho na gestão de leitos.

\section{Metodologia}

Trata-se de um estudo descritivo, exploratório, de abordagem qualitativa, realizado por meio de análise de conteúdo (Bardin, 2011). A pesquisa qualitativa é aplicada tradicionalmente em uma abordagem interpretativa, e os significados se valem de diferentes estratégias de investigação (Creswell, 2014), de tal forma que, no constructo final, predomine a lógica dos atores em sua diversidade e não apenas as suas falas, dentro de uma narrativa teorizada, contextualizada, concisa e clara (Minayo, 2012).

A pesquisa foi realizada com enfermeiros do Distrito Federal que atuam na área hospitalar, sendo enfermeiros que atuam na Gestão de Leitos (GL) e enfermeiros que atuam nas unidades assistenciais. Os participantes foram convidados a responder um instrumento de coleta de dados elaborado no Google Forms. Participaram do estudo 8 enfermeiros da gestão de leitos, 8 enfermeiros das unidades assistenciais e 1 enfermeiro que atua simultaneamente na GL e em unidade assistencial, totalizando 17 participantes.

Foram definidos como critérios de inclusão: ser enfermeiro, atuar nas unidades assistenciais e/ou atuar na Gestão de Leitos; aceitar participar da pesquisa; ter experiência superior a 6 meses na atenção hospitalar e/ou gestão de leitos. Como critérios de exclusão: não responder completamente o instrumento de coleta de dados; atuar há menos de 6 meses na assistência hospitalar e/ou na gestão de leitos.

A identificação dos enfermeiros foi feita por meio da técnica Bola de Neve em Redes Sociais Virtuais, conforme descrito por Costa (2018). A técnica consistiu em identificar inicialmente um pequeno grupo de participantes com as características que os membros da amostra deveriam ter. 
$\mathrm{Na}$ sequência, apresentou-se a proposta do estudo e, após obter/registrar tais dados, solicitou-se que os participantes da pesquisa indicassem outras pessoas pertencentes à mesma população-alvo. Esse processo continuou até que as métricas estabelecidas antecipadamente para a coleta de dados, como prazo de coleta ou quantidade máxima de entrevistados, foram atingidas: uma forma de amostra não probabilística que utiliza cadeias de referência (Costa, 2018).

A coleta de dados ocorreu no período de 22 de agosto a 21 de outubro de 2020 . O instrumento de coleta de dados foi adaptado do estudo realizado por Borges, Bernardino, Stegani e Tonini (2020). O instrumento, baseado no objetivo proposto, abordou a seguinte pauta: como o trabalho realizado pelo enfermeiro pode contribuir para o aumento da rotatividade dos leitos e a redução do tempo de permanência; qual a composição ideal da equipe e atividades desenvolvidas; como ocorre a tomada de decisão para a internação de pacientes nos leitos disponíveis; a qualificação e experiência exigida para o enfermeiro da GL; dificuldades e facilidades encontradas; interface entre os serviços; e resultados do trabalho mediante atuação do enfermeiro nessa área. A decisão por encerrar a recepção de novos instrumentos preenchidos se baseou na saturação dos dados (Flick, 2009) e no esgotamento do prazo destinado à coleta de dados.

Os dados foram analisados por meio da análise de conteúdo, que ocorreu em três etapas: a primeira etapa foi realizada por meio de leitura flutuante de todo o material transcrito, de forma a permitir apreender e organizar aspectos importantes para as fases seguintes da análise; a segunda etapa consistiu na exploração do material e no agrupamento do texto em categorias pré-estabelecidas; e a terceira etapa, o tratamento e a interpretação dos dados, permitiu a realização de inferências dos conteúdos obtidos na entrevista (Bardin, 2011).

Dessa análise foi possível encontrar três núcleos de sentido. Esses núcleos foram divididos em 3 categorias e 10 subcategorias: categoria 1 - Perfil da equipe da gestão de leitos segundo a qualificação e experiência profissional (composição da equipe; qualificação profissional); categoria 2 - Atuação do enfermeiro na Gestão de Leitos (atividades desenvolvidas; tomada de decisão; interface com os serviços; otimização dos leitos; continuidade do trabalho; resultados da atuação do enfermeiro); categoria 3 Dificuldades e facilidades nos processos de trabalho da Gestão de Leitos (dificuldades enfrentadas; potencialidades visualizadas).

Este estudo seguiu as determinações da Resolução no 466, de 12 de dezembro de 2012 , do Conselho Nacional de Saúde, que dispõe sobre diretrizes e normas regulamentadoras de pesquisas envolvendo seres humanos. O projeto foi aprovado pelo Comitê de Ética em Pesquisa da Universidade Católica de Brasília - UCB sob o Parecer № 4.209.913, de 12 de agosto de 2020.

\section{Resultados}

Foram entrevistados, por meio do Google Forms, 17 enfermeiros, sendo 8 que atuavam na assistência, 8 na gestão de leitos e 1 deles em ambas as atividades. Dentre os participantes, 58,8\% apresentaram especialização como maior titulação, com média de tempo de atuação de 2,5 anos, sendo que $29 \%$ têm menos de 1 ano de atuação e $35 \%$ contam com mais de 10 anos na gestão de leitos, conforme Tabela 1.

Tabela 1. Perfil dos entrevistados por titulação, área, setor e tempo de atuação. Brasília-DF, 2020.

\begin{tabular}{lcc}
\multicolumn{1}{c}{ CARACTERÍSTICAS } & $\mathbf{F}$ & $\%$ \\
\hline TITULAÇÃO & & \\
Doutorado & 2 & 11,8 \\
Mestrado & 1 & 5,9 \\
Especialização & 10 & 58,8 \\
\hline
\end{tabular}




\begin{tabular}{|c|c|c|}
\hline CARACTERISTICAS & $\mathbf{F}$ & $\%$ \\
\hline Graduação & 4 & 23,5 \\
\hline \multicolumn{3}{|l|}{ Qual sua área de atuação? } \\
\hline Enfermeiro assistencial & 8 & 47,1 \\
\hline Enfermeiro assistencial, Gestor de leitos & 1 & 5,9 \\
\hline Gestor de leitos & 8 & 47,1 \\
\hline \multicolumn{3}{|l|}{ Qual seu setor de atuação? } \\
\hline Gestão de leitos & 7 & 41,2 \\
\hline Outro setor & 8 & 47,1 \\
\hline Pediatria & 1 & 5,9 \\
\hline UTI adulto & 1 & 5,9 \\
\hline \multicolumn{3}{|l|}{ Área da pós-graduação } \\
\hline Ciências da Saúde & 2 & 11,8 \\
\hline Enfermagem & 1 & 5,9 \\
\hline Enfermagem do trabalho & 2 & 11,8 \\
\hline Epidemiologia & 1 & 5,9 \\
\hline Gestão em Saúde & 3 & 17,6 \\
\hline Gestão hospitalar & 1 & 5,9 \\
\hline Outras & 3 & 17,6 \\
\hline Terapia Intensiva & 2 & 11,8 \\
\hline Urgência e Emergência & 2 & 11,8 \\
\hline \multicolumn{3}{|l|}{ Tempo de atuação } \\
\hline Menos de 1 ano & 5 & 29,4 \\
\hline 2 a 5 anos & 3 & 17,6 \\
\hline 6 a 10 anos & 3 & 17,6 \\
\hline Mais de 10 anos & 6 & 35,3 \\
\hline Total & 17 & 100,0 \\
\hline
\end{tabular}

A partir dos relatos dos entrevistados, foi possível encontrar 3 núcleos de sentido que foram divididos em 3 categorias e 10 subcategorias, permitindo fundamentar as interpretações feitas na discussão e interpretá-las de acordo com a produção científica atualizada, bem como com as políticas públicas referentes a atenção hospitalar.

Quadro 1. Categoria 1 - Perfil da equipe de gestão de leitos segundo qualificação e experiência profissional. Brasília-DF, 2020.

\begin{tabular}{ll}
\hline Subcategorias & \multicolumn{1}{c}{ Unidades de Registro } \\
\hline $\begin{array}{l}\text { Composição } \\
\text { da equipe }\end{array}$ & $\begin{array}{l}\text { Composição mínima: médico, enfermeiro, técnico em enfermagem e } \\
\text { técnico administrativo, com ao menos } 1 \text { servidor de cada categoria } \\
\text { profissional (17) } \\
\text { Conhecimento de Gestão (10) e da assistência (3) gestão de conflitos (2) } \\
\text { gestão em saúde (1); Interesse em aprender e saber lidar com diferentes } \\
\text { processos de trabalho (2); Desenvolver habilidades para interação e } \\
\text { profissional } \\
\text { articulação com as categorias profissionais dos serviços intra e extra- } \\
\text { hospitalares (3); Estar apto a manipular os sistemas de informação; Ser } \\
\text { proativo, ter conhecimento do funcionamento dos setores do hospital (1); }\end{array}$ \\
\hline
\end{tabular}


A primeira categoria revelou aspectos referentes a composição da equipe e qualificação/experiência sugerida para o enfermeiro da gestão de leitos. Observou-se que não se trata de um trabalho individual, mas um trabalho em equipe.

Quadro 2. Categoria 2 - Atuação do enfermeiro na Gestão de Leitos. Brasília-DF, 2020.

$\begin{array}{ll}\text { Subcategorias } & \text { Unidades de Registro }\end{array}$

Atividades

desenvolvidas

decisão

Interface com

os serviços

Otimização dos

leitos

Continuidade

do trabalho

Resultados da

atuação do

enfermeiro
Levantamento (busca ativa) dos leitos disponíveis em todas as unidades de internação (12); Direcionamento dos pacientes aos leitos vagos (5); Aplicar a metodologia KANBAN (3); Elaborar medidas gerenciais para atuar nas pendências que impactem no aumento do tempo de permanência (3); Monitorar a inserção e autorização dos exames no SISREG (3); Utilizar o SISLEITOS para regulação de leitos intra e inter-hospitalar (3); Elaborar planilhas de indicadores (taxa de ocupação e tempo médio de permanência e giro de leitos (3); Atuar no acompanhamento das internações e altas (3) e nas transferências internas e externas (3).

A tomada de decisão ocorre pela avaliação do quadro clínico do paciente (9); Alocado conforme a disponibilidade de leitos (9); Seguem critérios de prioridade: egressos de UTI, centro cirúrgico-urgência, alta de box e sala vermelha, PS, eletivos (7); Via SISLEITOS e avaliação do quadro clínico pelo Médico (1); A tomada de decisão é feita com os gestores envolvidos (1).

A interface entre as equipes é boa (3); Por vezes complicada (1) e conflituosa em alguns momentos (1); Vivemos apagando incêndio, criando leitos onde fisicamente não podem existir (1); A comunicação é direta com visitas às unidades assistenciais, ou por telefone ou mesmo por aplicativo de mensagens (2).

Identificação, monitoramento e resolução das pendências (8); Garantir uma assistência de qualidade (4), segura e livre de danos (2), diminui o tempo de internação, aumentando a rotatividade dos leitos; Utilização de plano terapêutico singular (PTS) envolvendo os profissionais que podem contribuir para a solução da demanda que está segurando o paciente no leito (1).

O enfermeiro contribui fazendo a contra-referência do paciente para a área de abrangência para a continuidade do cuidado, evitando reinternações precoces (3); Atuando na utilização dos fluxos entre as unidades de internação e entre os diferentes serviços de saúde (2).

Foi observado redução do tempo médio de permanência do paciente (6); Maior rotatividade dos leitos (4); Desafogamento da emergência/pronto socorro (2); Melhora da comunicação (1); Transparência (1); Desvinculação do leito hospitalar ao profissional médico (1); Percebido que muito ainda precisa ser feito, e conquistado nosso espaço todos os dias (1). 
A segunda categoria mostra aspectos relacionados ao resultado da atuação do enfermeiro na Gestão de Leitos: atividades desenvolvidas, tomada de decisão, interface com os serviços, indicadores tais como taxa de ocupação, tempo médio de permanência, otimização dos leitos e trabalho do enfermeiro para a continuidade do cuidado.

Quadro 3. Categoria 3 - Dificuldades e potencialidades nos processos de trabalho da Gestão de Leitos. Brasília-DF, 2020.

\begin{tabular}{ll}
\hline Subcategorias & Unidades de Registro \\
\hline & Uma das maiores dificuldades é a ausência de leitos em bom estado \\
& disponível para alocar os pacientes (8); Fragilidade na comunicação com \\
& as unidades de internação (8) caracterizada por enfrentamento com \\
& enfermeiro do plantão para admissão de pacientes sem condições clínicas \\
& para serem mantidos em enfermarias; Falta de RH (4) com sobrecarga e \\
Dificuldades & acúmulo de competências; Falta de gestores capacitados para atuar na \\
enfrentadas & área da gestão de leitos (2); Resgatar os egressos de UTI dentro do tempo \\
& preconizado pelas portarias (2); Falta de médicos para discussão dos \\
& casos clínicos (2); Desconhecimento pela equipe das unidades \\
& assistenciais do papel desenvolvido pela Gestão de Leitos (2). \\
& Conhecimento do perfil clínico do paciente e a visão de cuidado (4); Agilizar \\
& o processo de trabalho (3) relacionado a diminuir o tempo de espera das \\
& cirurgias dos pacientes internados, diminuir o tempo de espera para \\
& procedimentos ambulatoriais, agilizar transferência para as Unidades de \\
Internação do Hospital e/ou outras Unidades Hospitalares da Retaguarda; \\
reduzir o número de pacientes na Emergência; Informatização dos \\
processos assistenciais e administrativos (2); Cumprimento dos protocolos \\
visualizadas \\
assistenciais relacionados a gestão de leitos (1).
\end{tabular}

A terceira categoria mostra aspectos relacionados às dificuldades enfrentadas e às potencialidades visualizadas nos processos de trabalho da gestão de leitos.

\section{Discussão}

A multifuncionalidade, característica da formação em enfermagem, possibilita a expansão dos espaços de atuação dos profissionais em todos os processos e procedimentos do SUS, inclusive na gestão, coordenação de programas, gerenciamento de leitos e assistência (Silva \& Machado, 2020). Sendo assim, a qualificação necessária para atuar na gestão de leitos, segundo os respondentes, deve estar atrelada ao conhecimento sobre gestão e assistência, proatividade, conhecimento do funcionamento do hospital, e interação/articulação com as categorias profissionais intra-hospitalar e com os demais serviços da rede assistencial.

Em consonância com tais dados, Borges, Bernardino, Stegani e Tonini (2020) ressaltaram que para gerenciar sistemas de saúde e relacionar-se com as equipes de saúde, há uma necessidade de enfermeiros altamente qualificados. Além do conhecimento na gestão e na assistência, o enfermeiro deve apresentar habilidades como liderança, tomada de decisão, trabalho em equipe, flexibilidade e negociação, comunicação e agilidade.

A atuação do enfermeiro, segundo os respondentes, está relacionada a processos sobre tomada de decisão, interface com os serviços, otimização dos leitos, elaboração de medidas gerenciais para a resolutividade das pendências e, em menor escala, ações que favoreçam a continuidade do cuidado pós-alta hospitalar. 
De maneira geral, a gestão de leitos exerce a função de estabelecer e monitorar os indicadores hospitalares, subsidiar discussões a respeito do perfil dos leitos hospitalares, fazer uso racional dos recursos hospitalares, praticar gestão das clínicas, acionar a rede de atenção à saúde para promover cuidado integral ao paciente e propor e atualizar os protocolos clínicos, terapêuticos e administrativos (Brasil, 2017).

Atualmente, o gerenciamento de leitos é considerado uma ferramenta importante de aprimoramento do fluxo do paciente, podendo ser executada por médicos e enfermeiros, com caráter estratégico e operacional. O manejo de leitos realizado por estes profissionais consiste em organizar a alocação de novas admissões para leitos vagos, por meio do conhecimento em tempo real do censo hospitalar e das demandas por internação (Soares, 2017)

A tomada de decisão, conforme foi colocado, ocorre pela avaliação do quadro clínico do paciente, atendendo aos critérios de prioridade, e com o apoio dos gestores envolvidos. Estudo semelhante realizado em hospitais públicos do DF revelou que a tomada de decisão ainda acontece a partir das relações de poder existentes no local, e que sofre influência do modelo assistencial vigente, no caso, o modelo biomédico, centrado na figura do médico como o principal tomador das decisões (Oliveira \& Bittencourt, 2020).

A interface entre as equipes das unidades assistenciais foi entendida como boa por alguns e como complicada e conflituosa por outros, cursando com estresse, revelado nas falas dos respondentes: "Vivemos apagando incêndio, criando leitos onde fisicamente não podem existir". Considerando-se o cenário do serviço público, de pouca oferta para grande demanda, embora a decisão de admitir e tratar pacientes seja responsabilidade dos médicos, são os enfermeiros os principais responsáveis pelo gerenciamento da capacidade total dos leitos (Borges, Bernardino, Stegani \& Tonini, 2020).

Sendo a média de internação variável, os gestores precisam de previsões adequadas sobre tempo médio de permanência e taxa de ocupação (Raffa, Malik \& Pinochet, 2017). Indicadores de produtividade são consideráveis no gerenciamento de leitos e quando se tem gestão eficaz, os leitos giram mais rapidamente e, por consequência, a taxa de ocupação e o tempo médio de permanência permanecem dentro dos padrões estipulados (Grala, Araújo \& Guerreiro, 2020).

De acordo com a Agência Nacional de Saúde Suplementar, o tempo médio de permanência (TMP) ideal para hospitais de grande porte é de 4 a 5 dias, e a taxa de ocupação (TO) entre $80 \%$ e $85 \%$. Um TMP maior que 7 dias está relacionado a um maior risco de infecção hospitalar. Uma TO abaixo de $75 \%$ indica baixa utilização e ineficiência na gestão do hospital. A utilização inadequada desse recurso pode indicar falha no planejamento do hospital ou inadequada articulação com a rede de serviços (Oliveira \& Bittencourt, 2020).

A contra-referência dos pacientes pós-alta hospitalar para a área de abrangência foi apontada como uma das ações desenvolvidas no intuito de promover a continuidade do cuidado e a desospitalização precoce segura. A presença do enfermeiro na equipe esteve relacionada a maior rotatividade dos leitos, desafogamento da emergência, transparência no uso dos recursos e desvinculação do leito hospitalar ao profissional médico, corroborando os achados de Borges, Bernardino, Stegani e Tonini (2020). Esses autores apresentaram uma série de bons resultados, os quais caracterizaram a performance do enfermeiro como uma "ponte" entre o leito hospitalar e o paciente.

Os aspectos evidenciados sobre as dificuldades enfrentadas estavam relacionados principalmente a falta de leitos disponíveis e funcionantes para alocar os pacientes e a fragilidade na comunicação com as unidades assistenciais, caracterizadas principalmente por enfrentamento com o enfermeiro do plantão no processo de admissão/alocação dos pacientes. Estudo similar aponta que o número de leitos disponíveis para a população está diminuindo no mundo todo, de forma que os leitos existentes merecem atenção especial para que se consiga, por meio deles, melhores resultados, seja do ponto de vista assistencial ou financeiro. 
O estudo também relatou dificuldades de integração entre as unidades, problemas de comunicação e busca de objetivos dissociados, que podem não contribuir para o alcance de bons resultados nos processos de atendimento (Raffa, Malik \& Pinochet, 2017).

O atraso na liberação dos leitos corrobora o atraso na admissão de pacientes, na alocação dos mesmos em leitos inapropriados (clínico versus cirúrgico, feminino versus masculino, utilização de isolamento sem indicação), no cancelamento de cirurgias eletivas, na demora de transferência de pacientes entre as unidades, no resgate de pacientes egressos de UTI, podendo elevar o tempo médio de permanência, expondo o paciente a eventos adversos evitáveis, como infecção, além de aumentar os custos da instituição e reduzir a qualidade da assistência (Castro Machado \& Machado, 2019).

Algumas potencialidades que envolvem o processo de fazer gestão de leitos foram consideradas pelos enfermeiros, tais como: o conhecimento adequado do perfil clínico do paciente e a visão integral do cuidado; a informatização dos processos assistenciais e administrativos por meio do SISREG, SISLEITOS, trakcare, entre outros. O principal objetivo de um sistema de informação é apoiar e provocar mudanças na organização para melhorar o funcionamento dos processos de trabalho e o cuidado da saúde. Esses sistemas têm se configurado como instrumentos que podem proporcionar muitas contribuições ao setor Saúde (Cavalcante, Ferreira \& Silva, 2011).

O conhecimento adequado do perfil clínico do paciente e a visão integral do cuidado, como potencialidades identificadas, são características intrínsecas da enfermagem e, mesmo com todos os avanços tecnológicos, essas habilidades, inerentes à profissão, assegurarão o lugar da Enfermagem em uma sociedade dominada pela tecnologia e pelo progresso científico. No entanto, para Fernandes, Esteves, Teixeira e Gherardi-Donato (2018), é necessário que a categoria reconheça seu próprio valor, tenha consciência das mudanças e cultive as características que legitimam a nossa humanidade, como relacionamento interpessoal, criatividade, presença e atenção, acolhimento e escuta, compaixão e capacidade contínua de aprendizado.

\section{Conclusões}

O gerenciamento dos leitos é uma atividade recentemente implantada nos hospitais públicos e privados e configura-se num campo de atuação em que a enfermagem vem ganhando espaço para agir de forma multidisciplinar, integrada a outras áreas e com liderança. Constitui-se como um serviço complexo que envolve todo o hospital e a rede de atenção à saúde num processo de trabalho que vai da admissão à desospitalização do paciente.

Este estudo identificou 3 categorias e 10 subcategorias relacionadas e interligadas entre si quanto ao processo da gestão dos leitos hospitalares. Na primeira categoria, evidenciaram-se aspectos importantes relacionados à composição e à qualificação da equipe. Na segunda categoria, ressaltou-se a importância da expertise em gestão e assistência, no entanto, foi apontado o interesse do profissional em querer aprender os diferentes processos de trabalho, visto que, devido à carência de recursos humanos nos serviços públicos, esses núcleos têm recebido um quantitativo maior de servidores sob restrição médica com a justificativa de tratar-se de serviço administrativo.

Destacou-se, também, a atuação da equipe, e principalmente do enfermeiro, diante dos enfrentamentos diários para equilibrar a relação entre a demanda e a oferta pelos serviços hospitalares. Evidenciou-se, ainda, a transparência dos processos, o desconhecimento da equipe assistencial sobre a autonomia que deve ter o time da gestão no gerenciamento dos leitos e a desvinculação do leito hospitalar ao profissional médico.

E, por último, na terceira categoria, quanto à atuação do enfermeiro na gestão de leitos, foram observadas potencialidades e dificuldades. Tais dificuldades estão relacionadas tanto à falta de leitos disponíveis e funcionantes como à comunicação entre as unidades assistenciais. 
Essa se configura como uma área em expansão que requer regulamentação, aperfeiçoamento e apoio da alta gestão no desenho e gerenciamento dos fluxos internos e externos nos diversos pontos de atenção à saúde.

O estudo teve como limitação a ausência de interação entre pesquisadores e participantes, por ter sido uma coleta de dados por meio de redes sociais. Algumas respostas descritivas foram difíceis de serem interpretadas. Também não foi devolvido aos participantes o consolidado das informações qualitativas para confirmar as interpretações, devido à limitação no cronograma. Estas limitações sugerem que o desenho de pesquisas qualitativas que se utilizam das redes sociais deva possibilitar mais de uma entrevista.

\section{Referências}

Bardin, L. (2011). Análise de Conteúdo. Edições 70.

Borges, F., Bernardino, E., Stegani, M. M., \& Tonini, N. S. (2020). Atuação de enfermeiros na gestão de leitos de um hospital de ensino. Revista Brasileira de Enfermagem, 73(4), 1-8.

Brasil. Lei no 7.498, de 25 de junho de 1986. Dispõe sobre a regulamentação do exercício da enfermagem e dá outras providências. Diário Oficial [da] República Federativa do Brasil, 26, 9273-9275.

Brasil. Ministério da Saúde. (2013). Portaria № 3.390, de 30 de dezembro de 2013. Institui a Política Nacional de Atenção Hospitalar (PNHOSP) no âmbito do Sistema Único de Saúde (SUS), estabelecendo-se as diretrizes para a organização do componente hospitalar da Rede de Atenção à Saúde (RAS). Brasília: Ministério da Saúde. Diário Oficial [da] República Federativa do Brasil, 253, Seção 1, 54-56.

Brasil. Ministério da Saúde. Secretaria de Atenção à Saúde. Departamento de Atenção Hospitalar e de Urgência. (2017). Manual de implantação e implementação: Núcleo Interno de Regulação para Hospitais Gerais e Especializados. Ministério da Saúde.

Castro Machado, D., \& Machado, A. C. A. (2019). A otimização do processo de gerenciamento de leitos e alta hospitalar. Saúde Coletiva (Barueri), 9(50), 1866-1872.

Cavalcante, R. B., Ferreira, M. N., \& Silva, P.C. (2011). Sistemas de Informação em Saúde: Possibilidades e desafios. Revista de Enfermagem da UFSM, 1(2), 290-299.

Costa, B. R. L. (2018). Bola de neve virtual: O uso das redes sociais virtuais no processo de coleta de dados de uma pesquisa científica. Revista Interdisciplinar de Gestão Social, 7(1), p. 1537.

Creswell, J. W. (2014). Investigação Qualitativa e Projeto de Pesquisa: Escolhendo entre Cinco Abordagens. Penso.

Fernandes, M. N. F., Esteves, R. B., Teixeira, C. A. B., \& Gherardi-Donato, E. C. S. (2018). O presente e o futuro da Enfermagem no Admirável Mundo Novo. Revista da Escola de Enfermagem da USP, 52, e03356.

Flick, U. (2009). Qualidade na pesquisa qualitativa. Artmed.

Fundação Oswaldo Cruz. (2019). Boletim Informativo do Projeto Avaliação do Desempenho do Sistema de Saúde (PROADESS), 4, fev./2019. Monitoramento da assistência hospitalar no Brasil (2009-2017).

Grala, A. P. D. P., Araújo, A. C., \& Guerreiro, P. O. (2020). Taxa de ocupação e média de permanência em quatro hospitais de um município sul-brasileiro. J. Nurs. Health, 2010300120103001

Minayo, M. C. D. S. (2012). Análise qualitativa: Teoria, passos e fidedignidade. Ciência \& Saúde Coletiva, 17, 621-626.

Miranda, G. M. D., Mendes, A. C. G., \& Silva, A. L. A. (2017). O desafio da organização do Sistema Único de Saúde universal e resolutivo no pacto federativo brasileiro. Saúde e Sociedade, 26, 329-335. 
Oliveira, B. P., \& Bittencourt, R. J. (2020). Avaliação por triangulação de métodos de um núcleo de gestão de leitos em um hospital público do distrito federal. Revista de Gestão em Sistemas de Saúde, 9(3), 406-432.

Raffa, C., Malik, A. M., \& Pinochet, L. H. C. (2017). O desafio de mapear variáveis na gestão de leitos em organizações hospitalares privadas. Revista de Gestão em Sistemas de Saúde, 6(2), 124-141.

Silva, M. C. N., \& Machado, M. H. (2020). Sistema de Saúde e Trabalho: Desafios para a Enfermagem no Brasil. Ciência \& Saúde Coletiva, 25(1), 7-13.

Soares, V. S. (2017). Análise dos Núcleos Internos de Regulação hospitalares de uma capital. Einstein (São Paulo), 15(3), 339-343.

Wasgen, A. M., da Silva Terres, M., \& Machado, B. F. H. (2019). O impacto do gerenciamento de leitos na gestão hospitalar. Revista Hospitalidade 16(2), 31-49. 